\title{
Michael Handelsman, pionero en los estudios de la literatura afroecuatoriana
}

\author{
Michael Handelsman, a pioneer \\ in studying Afro-Ecuadorian Literature
}

\author{
María Auxiliadora Balladares \\ Universidad San Francisco de Quito, Ecuador
}

DOI: https://doi.org/10.32719/13900102.2019.45.5

Fecha de recepción: 15 enero 2019

Fecha de aceptación: 4 abril 2019 


\section{RESUMEN}

Este artículo revisa los aportes de Michael Handelsman a los estudios de la literatura afroecuatoriana. Handelsman es un pionero en el campo y su libro de 1999, Lo afro y la plurinacionalidad: el caso ecuatoriano visto desde su literatura, es el mayor aporte y el estudio más cuidadoso y profundo respecto de la importancia de observar, en el giro del siglo, los aportes de escritores afro en nuestro país, particularmente en un momento en el que el debate en torno a la plurinacionalidad es central. El objetivo principal del libro de Handelsman es pensar un Ecuador plurinacional desde el aporte de la comunidad afro y no solo desde la centralidad tradicionalmente otorgada a las comunidades indígenas. Para esto, el autor estudiará diferentes expresiones literarias, desde la percepción del sujeto afro en la literatura del Grupo de Guayaquil, pasando por la obra de los mayores escritores afroecuatorianos del siglo $\mathrm{XX}$, hasta las diferentes expresiones sobre lo afro vertidas en revistas culturales a lo largo del siglo pasado.

Palabras clave: Michael Handelsman, Ecuador, escritores, plurinacionalidad, literatura afroecuatoriana.

\section{ABSTRACT}

This article reviews Michael Handelsman's contributions to studies on Afro-Ecuadorian Literature. Handelsman is a pioneer in the field, and his book published in 1999, Lo afro y la plurinacionalidad: el caso ecuatoriano visto desde su literatura, is the most outstanding contribution and the most careful and profound study regarding the importance of observing, in the turn of the century, the contributions made by Afro-Ecuadorian writers, particularly in a moment in which the debate on pluri-nationality is crucial. The main objective of Handelsman's book is to think a pluri-national Ecuador from the Afro-Ecuadorian community's production, and not just from the centrality as traditionally assigned to Indigenous communities. For this, the author will study different literary expressions, from the perception of the Afro subject in the works produced by the Guayaquil Group, going through the works of the most outstanding Afro-Ecuadorian writers of the $20^{\text {th }}$ Century, until reaching the diverse expressions about the Afro, expressed in cultural magazines throughout the past century.

KEYwORDS: Michael Handelsman, Ecuador, writers, pluri-nationality, Afro-Ecuadorian Literature.

LOS FINALES DE los 1990 es el momento en el quisiera concentrarme para plantear un primer acercamiento respecto de los estudios de la literatura afroecuatoriana a manos de Michael Handelsman. No porque antes no haya habido en su prolífica obra un acercamiento tanto a la literatura como a las comunidades e intelectualidad afro de nuestro país, sino porque en el año 1998 se escribe una nueva constitución del Ecuador que define al Estado ecuatoriano como pluricultural y multiétnico y un año 
después, en 1999, Handelsman publica el libro Lo afro y la plurinacionalidad: el caso ecuatoriano visto desde su literatura, que es uno de los más importantes aportes al estudio de esta literatura y que, desde la tradición de los estudios culturales, propicia y aporta a un necesario debate en torno al rol de la comunidad afro como parte de un Ecuador plurinacional. Lo plurinacional como categoría para pensar al país surge en el contexto de las luchas indígenas de los ochentas y noventas. En el debate de esas décadas, la comunidad afro permanecía relegada respecto de la centralidad que tradicionalmente en nuestro país se le ha otorgado a las comunidades indígenas. En ese sentido es que el libro de Handelsman apunta a pensar en la importancia de la comunidad afroecuatoriana en el panorama de la plurinacionalidad. Su estudio de las expresiones literarias y culturales afro se alinea con una postura que, desde la perspectiva de analistas constitucionales como Agustín Grijalva (2009), es mucho más enfática en lo político y la interacción cultural, antes que con la perspectiva culturalista y estática asumida, a pesar de los debates de esa década, en la Carta Magna del 98.

Hacia finales de los 1990, Michael Handelsman señalaba:

Aunque la tendencia de tratar el proyecto plurinacional casi exclusivamente en términos de los reclamos articulados por los indígenas, es esencial anotar que si dicho proyecto va a conducir a una nueva concepción y a una amplia aceptación de la heterogeneidad del país, urge reconocer plenamente la presencia afro en todo discurso plurinacional $(1999,5)$.

Esa presencia se evidencia a partir de un estudio concienzudo de la literatura en donde el afroecuatoriano es personaje (en particular en la obra del Grupo de Guayaquil y las crónicas de Jorge Martillo recopiladas en Viajando por pueblos costeños), así como en el acercamiento a la literatura de escritores afros (como Adalberto Ortiz, Nelson Estupiñán Bass, Antonio Preciado y Argentina Chiriboga) y en las publicaciones periódicas que a lo largo del siglo XX surgieron en la provincia de Esmeraldas para pensar la cultura, la situación socio-económica y sobre todo las implicaciones que el racismo y el abandono del Estado provocan sobre la conformación de la identidad afro, así como la necesidad de pensar desde una postura afrocéntrica buena parte de la producción literaria y cultural del siglo pasado. Su estudio atento se sostiene en buena medida en la obra de pensadores vinculados con los estudios de la cultura, de la subalternidad, así como teóricos poscoloniales como Frantz Fanon, Anderson Benedict, Kwame 
Anthony Appiah, Homi Bhabha y Néstor García Canclini; asimismo en el pensamiento de estudiosos de la sociedad y la cultura ecuatorianas como Justino Cornejo, Laura Hidalgo Alzamora, Humberto E. Robles, Juan García Salazar, entre otros. En el contexto ecuatoriano, el libro de Handelsman y el de Regina Harrison (Entre el tronar épico y el llanto elegíaco: simbología indigena en la poesía ecuatoriana de los siglos XIX y XX de 1996) son los dos aportes más importantes que ecuatorianistas estadounidenses han escrito hacia finales del siglo XX para rastrear históricamente las diferentes formas de producción literaria sobre y por el sujeto subalterno, así como las formas que adquiere su resistencia respecto de los proyectos modernizadores estatales que los excluyen. Handelsman, en concreto, identifica dos de las fuentes de esa exclusión: el mestizaje integracionista de corte vasconceliano $(1999,167)$ y el binarismo blanco-indio sobre el cual se ha montado la discusión en torno a la identidad ecuatoriana.

En lo que se refiere al siglo XX, Handelsman identifica las tres etapas por las que ha pasado el interés en lo negro: la primera, el negrismo de inicios de siglo, donde priman el exotismo y el primitivismo (el europeo regresa a ver a África en busca de musas); el segundo, la negritud de las décadas del 30 y el 40 que es un movimiento de intelectuales afroamericanos que busca reivindicar África y lo afro "como las bases de una civilización rica en tradiciones e historia y no un mero depósito de curiosidades" (Handelsman 1999, 159), y el tercero, el afrocentrismo, que implica un hacerse continuo que se opone a cualquier proyecto que quiera mantener lo negro en el plano de lo simbólico o del "folklore" (1999, 160). Esta tercera etapa coincide con el surgimiento del Proceso de Comunidades Negras en nuestro país, sobre el que volveré más adelante. Esta es, a los ojos de Handelsman, la etapa más valiosa en el sentido de que en ella se logra concretar un proyecto identitario que, sin negar las tensiones y las problemáticas de la comunidad afro, plantea una constante reivindicación de su cultura y su tradición, que rompe con los estereotipos y preconceptos racistas, tal como sostiene Juan García Salazar, quien en vida fuera uno de los más importantes dirigentes del pueblo negro ecuatoriano de las últimas décadas:

Para toda América Latina vinieron cerca de 11 millones de negros. Si los negros fuéramos vagos, ¿por qué se tomaron la molestia de traer 11 millones de negros a pasear a América Latina? Entonces, vaya si son vagos estos españoles. No vinimos de África a pasear, vinimos a trabajar y nosotros 
somos concientes que los capitales que formaron el patrimonio de estas naciones fueron el producto del trabajo de mano esclava (en Handelsman 1999, 163).

Esta cita, que es radical en su tono y contenido, refleja muy explícitamente el camino de la reivindicación. Un camino que, por un lado, exige reconocer, en la línea de Benjamin, la barbarie que ha significado el erigir los monumentos culturales de las naciones esclavistas (Benjamin 2005, 21-2), así como, en la línea de Fanon, el hecho de que la modernidad capitalista ha sido posible solamente gracias al trabajo esclavo (Fanon 2001). Handelsman defiende la centralidad de la presencia afroecuatoriana respecto de lo ecuatoriano y de lo andino: en esos imaginarios, sostiene, esta "ha sido marginada ya que se la ha considerado erróneamente como ancilar o exótica -como una cultura desplazada del Caribe" $(1999,5)$. En ese sentido, se refiere a cualquier aceptación del negro ecuatoriano en tanto componente integral del contexto nacional como un "simulacro" $(1999,14)$.

Cuando desde la sociedad civil y el Estado se guardó un silencio imperdonable, el aporte central de Handelsman ha sido ofrecer en un momento clave de la historia nacional una cartografía de la producción literaria de la negritud y la producción afrocéntrica para recordarnos la necesidad de replantear nuestros contenidos escolares, de ofrecer las herramientas adecuadas para que la nación reconozca la riqueza de las manifestaciones culturales afroesmeraldeñas, sus raíces, sus componentes identitarios.

El análisis de Handelsman parte por referirse a la literatura de la Generación del 30, en particular del Grupo de Guayaquil. Al respecto sostiene que "Su caracterización del negro pecaba a menudo de ser estereotípica y lamentablemente, la complejidad del drama vivido por los negros ecuatorianos corría el riesgo de perderse en una denuncia social que no contemplaba la raza más allá de su dimensión fenotípica” (1999, 27). El concepto de mestizaje al que algunos escritores de esta generación apelan, en buena medida, va a coartar la especificidad socio-cultural de los negros en el Ecuador. Los personajes negros de la literatura del Grupo de Guayaquil "no constituía una expresión de negritud o de lo afro, por lo menos en términos culturales e históricos más allá de la denuncia social o de lo exótico y lo primitivo" $(1999,38)$. Desde esta clara identificación, Handelsman lee los personajes negros más representativos de la narrativa 
de los 30: Alfredo Baldeón, Baldomera, Masa Blanca y Bulu-Bulu. En esta narrativa, no se evitan los estereotipos y cierta tendencia a folclorizar al personaje negro. Insiste en que se impone la necesidad de nuevas lecturas de nuestros clásicos, revisándolos desde una perspectiva afrocéntrica (así como desde otras, por ejemplo, la feminista) para determinar sus aportes, pero también sus vacíos e inconsistencias, que en el caso de los de Guayaquil, sería, sobre todo, no entender o entender a medias que "más que un accidente biológico, la raza supone vivencias culturales” (1999, 46). Los tres escritores a los que lee con más detenimiento son Aguilera Malta, De la Cuadra y Gil Gilbert. Aguilera Malta, que es, de los tres, quien más trabajó personajes negros y más se refirió a sus problemáticas sociales, es también quien sostuvo sin mayor empacho que no existe el racismo hacia los negros en el Ecuador. Este andar a caballo entre el reconocimiento solidario de la violencia racial hacia la población negra y su negación, se observa, para seguir con Aguilera, en el contrapunto entre sus declaraciones poco felices respecto de la ausencia del racismo y el hecho de que se haya interesado por hacer lecturas de pensadores revolucionarios negros tan importantes como Fanon y haberlos traducido al castellano.

En su lectura de la novela de Velasco Mackenzie, Tambores para una canción perdida de 1986, Handelsman reconoce dos movimientos significativos: el primero, romper con los esquemas que tradicionalmente han relegado lo afroecuatoriano a la provincia de Esmeraldas, y el segundo, contrarrestar "un falso mestizaje nacional" $(1999,82)$. Como corolario, el efecto central de la novela de Mackenzie ha sido obligar "al lector a revalorar el papel medular que ha jugado, y que sigue jugando, una herencia africana de muy diversos matices que vincula al Ecuador y, sobre todo, a la Costa ecuatoriana, a un circuito cultural panafricanista que a su vez incorpora, de una manera u otra, a toda América” (1999, 87). En esa misma línea, lee el aporte de las crónicas de viaje de Jorge Martillo por la costa ecuatoriana: la herencia afro se extiende por fuera de los territorios de Esmeraldas, de ahí que sus crónicas sobre Guayaquil y la provincia verde estén conectadas a partir de esa herencia compartida. Con su estudio de estas crónicas, Handelsman alcanza uno de sus objetivos centrales: “ampliar más el concepto de lo afroecuatoriano" (1999, 93), al observar cómo su incidencia se extiende a lo largo de toda la costa ecuatoriana y poner en entredicho el hecho de que Ecuador sea considerado, siempre y solamente, país andino. Se trata, pues, de entender cómo lo afro es menos 
un fenómeno racial y más uno cultural, de rastrear las sutilezas donde se instala la presencia africana.

Handelsman hace un recuento histórico del racismo predominante desde el comienzo de la República en los discursos y declaraciones de figuras trascendentales como Vicente Rocafuerte que desdeñará de la participación de negros y mulatos de Colombia y Venezuela catalogándolos como una amenaza para la seguridad nacional. Esta veta racista se extenderá hasta el siglo XX en la voz de cierta intelectualidad que mira con indiferencia y desdén a negros y mulatos. Cita a Alfredo Espinosa Tamayo quien en 1918 "descartó a los afroecuatorianos como miembros de 'una raza servil, creada en la esclavitud', y anunció su oposición inalterable a la mezcla de razas puesto que el africano era conocido por haber sido maldecido con 'cualidades mentales inferiores'” (1999, 107).

La segunda mitad de su libro, comienza con la lectura de la obra de los cuatro escritores afroecuatorianos mencionados anteriormente: Ortiz, Estupiñán Bass, Preciado y Chiriboga. En 1980, Estupiñán Bass reflexionó sobre la imperiosidad de repensar los conceptos de nacionalidad, mestizaje y negritud. Sobre este último, señaló la importancia de pensarlo en un contexto panamericano, atendiendo a la realidad ecuatoriana, ciertamente, pero sin descuidar su ser afro, reconociéndolo como parte constitutiva de su identidad. La asimilación de las llamadas minorías no se sostiene más en el contexto poscolonial de finales de siglo XX. Como diría Bhabha, a quien Handelsman lee con mucha atención y cuyo pensamiento roza con su propuesta: "El tiempo para asimilar minorías a nociones totalizadoras y orgánicas de valor cultural se ha acabado dramáticamente" (Bhabha en Handelsman 1999, 118). En su lectura de Juyungo de Ortiz, destaca el hecho de

haber marcado un primer paso en lo que García Canclini ha identificado como la reconstrucción de una nacionalidad abiertamente intercultural. El haber insertado lo afro en el escenario nacional ecuatoriano abrió fisuras en el andamiaje blanco-mestizo y, aunque el problema racial todavía está lejos de resolverse en el Ecuador, Ortiz logró remover muchas de las tensiones y fuerzas sociales que siguen deteniendo la construcción de un Estado unido a partir de sus múltiples particularidades (Handelsman 1999, 129).

De Jonatás y Manuela de Chiriboga destaca que la autora haya escogido a personajes mujeres y esclavas para buscar alcanzar un discurso pluricultural y así observar cómo la historia empieza a contarse desde los 
márgenes en donde se encuentran el afrocentrismo, el feminismo y la democratización cultural. Narrar la experiencia del esclavo significa, en el mismo gesto que ha leído John Beverley en el testimonio, poner el margen en el centro (Beverley 2010).

Finalmente, Handelsman lee el Proceso de Comunidades Negras ${ }^{1}$ como una respuesta contestaria frente a la tradición mestiza. Juan García insistía en que el Ecuador es un país racista y que para las comunidades negras es hora de "atrincherarse, apalencarse" (1999, 156). Los derechos reclamados en el texto que lleva por título "Algunos de los derechos que a los negros nos gustaría tener en la Constitución" firmado por el "Proceso de Comunidades Negras del Norte de Esmeraldas" son los derechos culturales [la etnoeducación], de participación, a la organización, al territorio, al desarrollo, al medio ambiente y los políticos. Se trata, en palabras de Michael, de ser actores dinámicos en lugar de receptores pacíficos en la construcción y en la evolución del país.

En su estudio de las revistas esmeraldeñas, Handelsman sostiene que escoge leer este particular corpus porque la producción y la escritura de una revista es un proyecto colectivo que se corresponde con la actitud de "apalencarse" de la comunidad afroesmeraldeña. Lee, en El Luchador (1905) el reclamo porque el esfuerzo vertido por los afroesmeraldeños en la causa liberal no se ha visto recompensado y porque las autoridades los han dejado en el olvido. En El Correo (1929), lee cómo el reclamo se enuncia con un evidente tono irónico. De Marimba (1935), menciona que los referentes culturales a los que hacía alusión la revista preparaban el terreno para otro tipo de discurso, el afroecuatoriano propiamente y señala la insistencia en que el abandono en el que se tiene a la provincia ocurre por una cuestión racial. De Hélice (1949), destaca su lema: "Por una cultura al servicio del Pueblo". De Tierra verde (1952), el hecho de que se trate de un órgano de la Casa de la Cultura Ecuatoriana, Núcleo de Esme-

1. El Proceso de Comunidades Negras es el "proceso organizativo e identitario que ha demandado al Estado nacional se le reconozca una serie de derechos reivindicativos políticos, económicos y culturales apelando a su pertinencia etno-cultural [...] su propuesta política y su necesidad de construir una identidad cultural se va a sustentar en símbolos ancestrales como el palenque, la territorialidad y las circunscripciones territoriales como máximos referentes de su propuesta política" (Patiño 2002, 10). ‘http://repositorio.uasb. edu.ec/bitstream/10644/2902/1/T0189-MEC-Pati\%C3\%B10-El\%20proceso.pdf.. Consulta: 17 de junio de 2018. 
raldas. Su primer director, César Névil Estupiñán, apunta, en los primeros años de la revista, a una incorporación de Esmeraldas a la vida nacional, dejando de lado la especificidad afro. Pasado el tiempo, sin embargo, el mismo Névil Estupiñán asume un discurso más radical y beligerante. Llega a plantear incluso el concepto de "hibridismo" en 1955, adelantándose al pensamiento poscolonial y muy en consonancia con lo que hoy llamamos plurinacionalidad. Finalmente, sobre Meridiano Negro (1980) "una revista cultural de los afroecuatorianos", menciona que lo afro no se matiza, sino que se entiende como esencial de la identidad esmeraldeña y es su estandarte de lucha (Handelsman 1999, 188). Destaca de las reflexiones vertidas en la revista el ver la migración interna de los afroesmeraldeños a las grandes ciudades como una forma de evidenciar su presencia en la vida nacional y la desaparición de toda vacilación en lo que respecta a pensar la raza como el estandarte de cualquier proyecto de reivindicación o de liberación. Junto a los intelectuales, destaca la participación del pueblo en esta revista. La cuidadosa lectura de archivo que hace Handelsman responde a la importancia que los órganos de publicación periódica van a tener en el Ecuador a lo largo de todo el siglo XX. Se trata del espacio en donde la intelectualidad ecuatoriana reflexionará sobre nociones identitarias en las que el tema de lo afro debió haber sido central y determinante.

Los aportes de Michael Handelsman al estudio de la literatura afroecuatoriana no solo nos remiten a los pormenores de una literatura poco atendida por nuestra intelectualidad y al diálogo fluido con el pensamiento más progresista de finales del XX, sino que, además, se deben entender como un gesto político trascendental en la constitución de un nuevo Estado, de una nueva sociedad civil que a partir de la lucha de los movimientos indígena, afro y feminista empieza lentamente a desprenderse de sus taras, a abandonar el anquilosamiento secular debido a los prejuicios de raza, clase y género. La obra de Handelsman insufla esperanza, que es el mayor de los magisterios posibles. *

\section{Bibliografía}

Benjamin, Walter. 2005. Tesis sobre la historia y otros fragmentos. México: Contrahistorias.

Beverley, John. 2010. "El margen al centro: sobre el testimonio". En Testimonio: sobre la politica de la verdad, 21-40. México: Bonilla Artigas Editores. 
KİPUS 45, enero-junio 2019

Fanon, Frantz. 2001. "La violencia". En Los condenados de la tierra, 30-98. México: Fondo de Cultura Económica.

Grijalva, Agustín. 16 de junio de 2009. "Principales innovaciones en la Constitución de Ecuador del 2008”. IRG. Disponible en 〈http://www.institut-ouver nance.org/es/analyse/fiche-analyse-454.html\#iref:6>. Consulta: 15 de junio de 2018 .

Handelsman, Michael. 1999. Lo afro y la plurinacionalidad: el caso ecuatoriano visto desde su literatura. Mississippi: Department of Modern Languages-The University of Mississippi.

Patiño Sánchez, Ninfa. 2002. El proceso de Comunidades Negras del Ecuador desde el testimonio de Juan García. Quito: Universidad Andina Simón Bolívar, Sede Ecuador. Disponible en 〈http://repositorio.uasb.edu.ec/bitstream/ 10644/2902/1/T0189-MEC-Pati\%C3\%Blo-El\%20proceso.pdf〉. Consulta: 17 de junio de 2018 . 